\section{Segmentation Before Quantization By Using Photoshop: Darkfield Images}

\author{
Jerry Sedgewick
}

University of Minnesota

sedge001@umn.edu

Adobe Photoshop may not come to mind as a program that is useful for segmenting (separating regions of interest from other visual information), but, in many ways, its functions can help scientists gain several end results. These include the ability to fully or partially automate the segmentation of entire directories of image files, the ability to better separate regions of interest from background, and the ability to narrow histograms (making the selection of a grayscale value less subjective when setting a threshold value).

These functions are especially useful when separating fluorescing objects from the background, something that is far more difficult than it appears. That's because our eyes tell us when scanning a fluorescent image from edge to edge that all the fluorescing objects are clearly brighter than background when, in fact, fluorescing objects often act like spotlights in fog so that background can be brighter near collections of fluorescing objects than within fluorescing objects which appear singularly. Add to that problems with uneven illumination across the field (when that uneven illumination is not corrected at acquisition), and the possibilities for segmenting or separating fluorescing objects from background for subsequent quantitation becomes a nightmare. But the problem can be overcome through the use of one image processing tool in Photoshop: the High Pass filter. The methods presented here are equally applicable to dark field images produced from material science specimens, for quantization of grain size, for example.

Before examining the use of that filter, it would be best to start from the beginning of a segmenting process. Here, then, is a series of steps commonly used for the segmenation of bright objects against a dark background (darkfield).

1. Duplicate your image so that you don't accidentally save over the original. When only a part of the image is needed for quantitation, outline area of interest with the lasso tool on the toolbar.

2. If the image is in color, separate color channels. If the image has been interpreted in color space (RGB color), then separate the colors into their individual red, green or blue channels. That can be accomplished by using Channe/ Mixer. In the menu, under Image select Adjust and then Channel Mixer. Choose the channel that correlates to the fluorophore you used, increase it to $100 \%$, and reduce the other two to $0 \%$ (e.g., if FITC was used, increase the green channel to $100 \%$ and reduce the red and blue to $0 \%$ ). If the fluorophore is unknown or the fluorescence results from auto- or bio-luminescence, choose the channel with the brightest fluorescence. If uncertainty still remains, the green channel is the default channel for grayscale images taken with conventional CCD chips. Put a checkmark next to Monochrome at the bottom of the Channel Mixer dialogue box.

Why not work with the color image rather than changing these to grayscale? While it's true that brighter colors can be selected versus the darker colors that make up background by using the Wand Tool (then selecting Similar under Select in the menu) or Color Range (also under Select), the experimentation necessary to arrive at good segmentation can take an entire afternoon, and quantitation programs typically need grayscale images for measurement anyway.

3. Change the mode of images formerly in RGB color to gray- scale. In the menu, under Image select Mode then Grayscale.

Note: Some files may appear to be grayscale, but are saved as indexed color, or as 16-bit files. Grayed out menu selections normally alert the user to either of these attributes. In the menu under Image, choose Mode and then Grayscale and/or 8-bits/Channel.

4 th or 5th step. Use the High Pass filter to separate bright objects from background. In the menu under Filter, select Other then High Pass. Drag the Radius slider until bright objects (which may now look more grayish) appear most separated from a flat background. If the radius is set too high, darkened areas appear under the brightest labeled objects. If it is set too low, objects are not clearly separated. It's better to err on the side of darkened areas under the brightest objects, since this image will eventually be thresholded. All images taken under the same conditions can subsequently be filtered at the same radius.

The High Pass filter appears to provide a separation of bright objects from background dependent upon the size of an adjustable "window" (the size set by adjusting Radius). When the window is smaller than the objects that are to be separated, no separation occurs. When the window is at the average size of the objects or larger, the window "sees" the entire objects as well as the backgrounds around the objects.

Note: This filter does wonders for phase contrast images. The morphology of cells are maintained while backgrounds are made completely even. You may also want to experiment with images in which DIC or Hoffman illumination has been used.

4. If necessary, use Unsharp Mask and most the time use Median Filter. If objects do not separate cleanly, you may want to experiment with sharpening by using Unsharp Mask. To reduce the noise that results from PMT's and CCD's, use the median filter.

For Sharpening. On the menu under Filter select Sharpen and then Unsharp Mask. Unsharp Mask provides darkening at edges of objects when the radius slider is set to greater dimensions, what is more important when using Unsharp Mask for segmenting (though this works best with an increase in Amount). The greater the darkening at the edges, the greater the ability to segment. Setting the radius too high, however, can introduce artifacts (it may cause

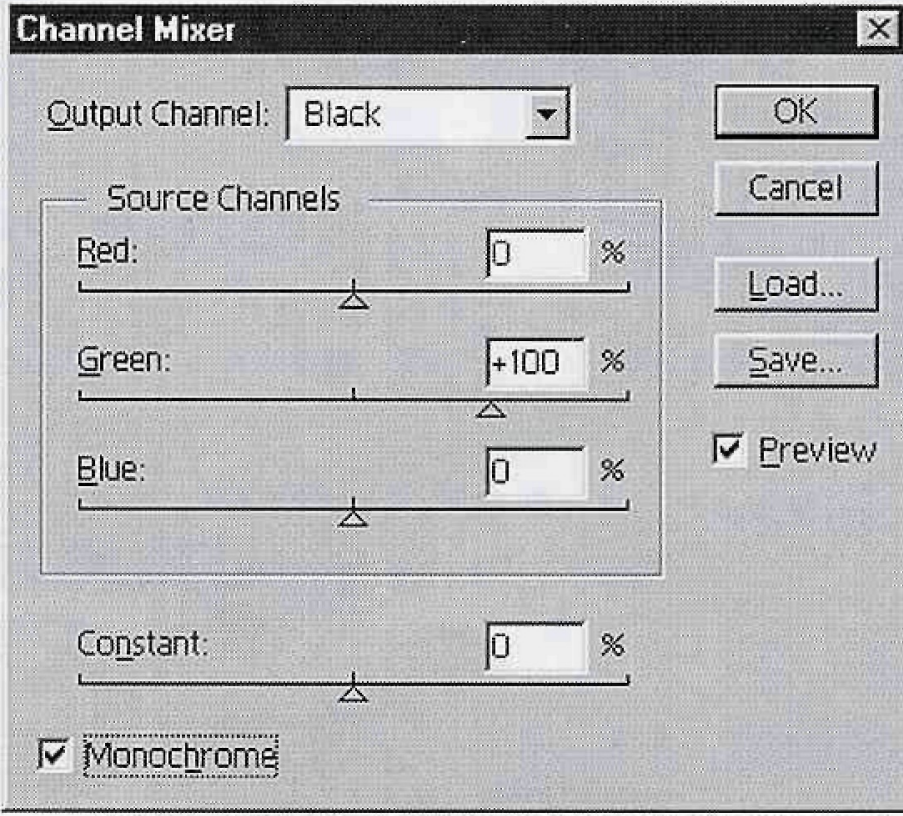

Figure 1. Example of settings used for extracting the green channel in the Channel Mixer dialogue box. 


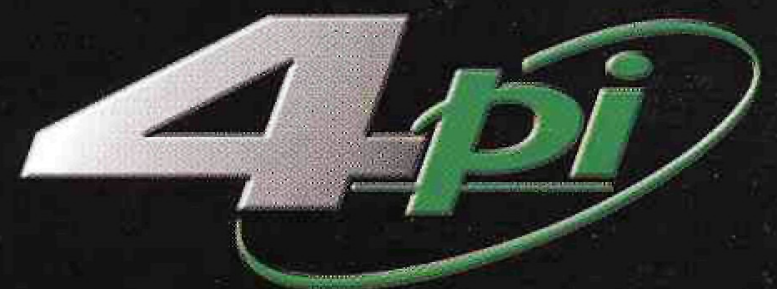

EDX \& Imaging Systems

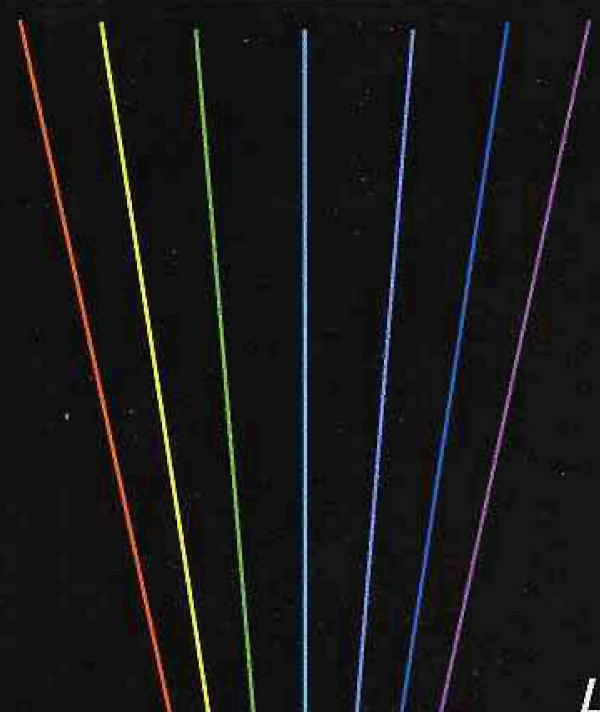

4pi Revolution ${ }^{\oplus}$ easy-to-use software

Light-element detection and quantitative analysis

Digital high-resolution EDX and image acquisition

Full range of $x$-ray detectors
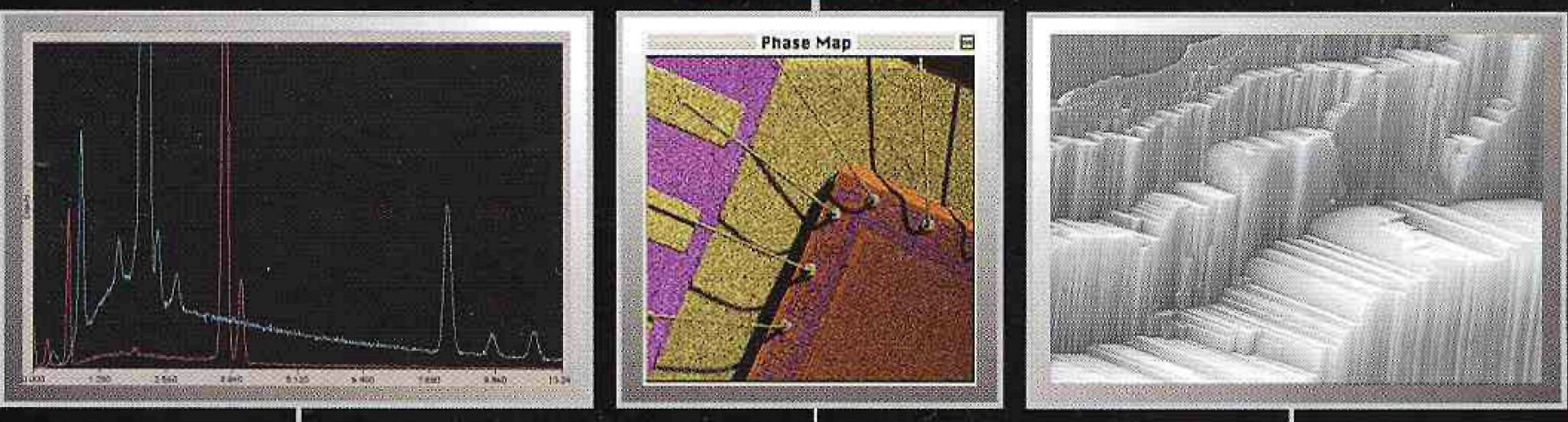
unwanted objects to separate along with the desired objects). The sharpening step normally occurs before using the high pass filter. This step may be unnecessary, depending upon how effectively bright objects segment (what is determined when the image is thresholded later on).

For Median Filtering. The median filter blurs details except at the edges. Thus, it maintains edges while flattening pixel intensities within objects and in the background. Because fluorescence by nature is dim, most CCD/PMT systems introduce noise, even with frame averaging and cooling. In the menu under Filter, select Noise and then Median. The slider is typically set at 1 except with visibly noisy images. Median filtering should be done after using Unsharp Mask and the high pass filter

5. Threshold the image. Now a grayscale value can be selected, below which all grayscale values will become absolute black, leaving the bright objects as pure white. In the menu, under Image choose Adjust and then Threshold. As the slider is moved away from the mean of the histogram, objects become smaller in dimension. The "correct" grayscale value setting for the slider now occurs about halfway down the slope of the histogram. The high pass filter has effectively narrowed the histogram to create a consistent slope and to make the selection of a value more objective. The edges and dimensions of the segmented objects will have to be compared with the original image to verify that the chosen setting is correct. Some of the dimmer fluorescing objects may drop out, but these may or may not have been counted manually, depending on
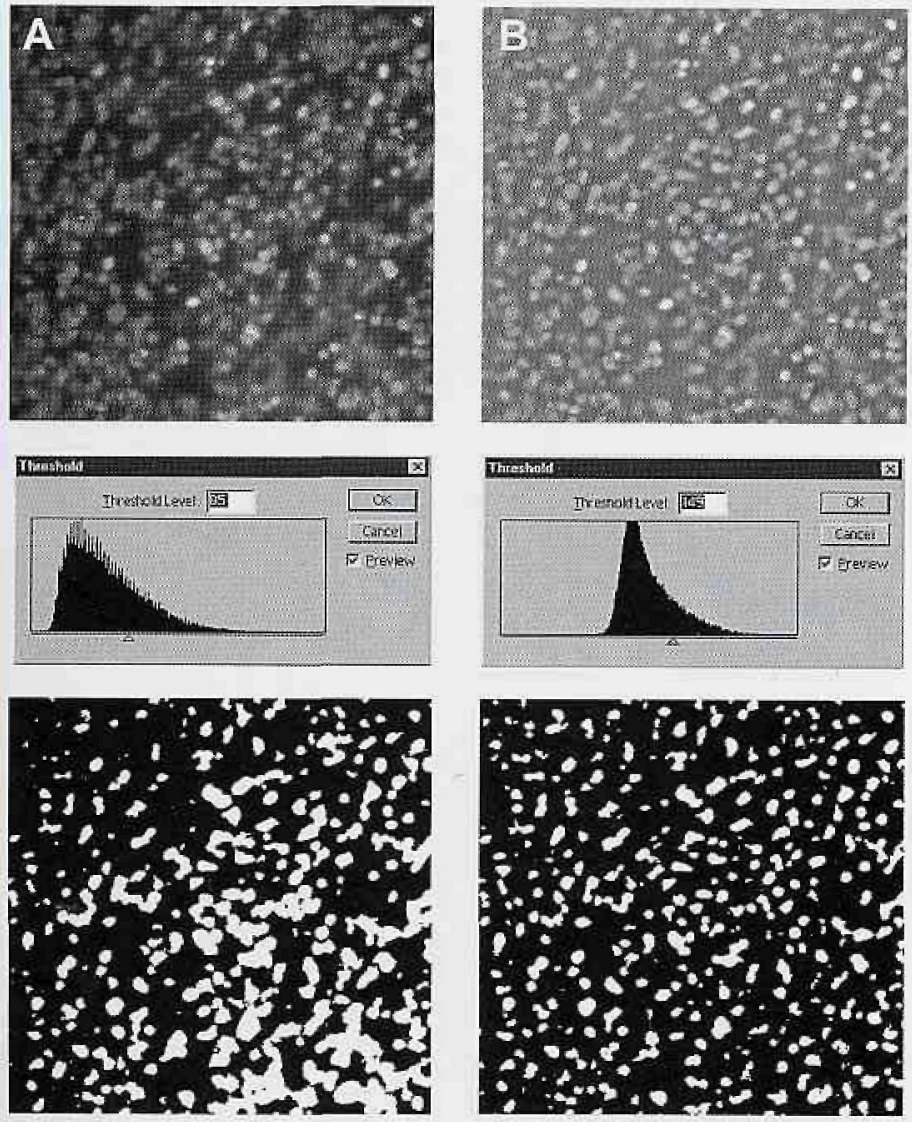

Figure 2. Column A shows what results from arriving at a threshold value from a grayscale image (top). Note the wide histogram (center) and the uneven segmentation of nuclei (bottom). Column B shows what results (bottom) from setting a threshold value after using the high pass filter on the same image (top). Note the evenness of segmentation from a less than optimal image, and the narrow histogram (center).

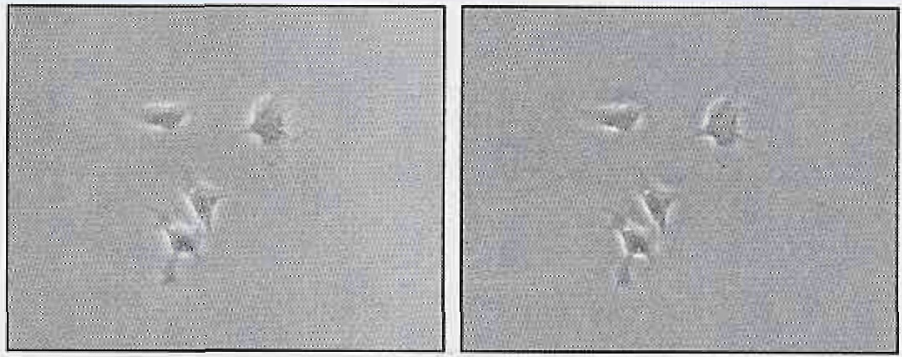

Figure 3. Uneven illumination when Illuminating with phase contrast (left). Background flattened on same image after using the high pass filter (right)

the human doing the counting. Fragments of fluorescing objects will have to be considered artifact since these cannot be positively identified. In either case, the use of stereology for quantitation effectively eliminates this kind of judgment, but that discussion is not within the scope of this article, for its use also eliminates the need for segmentation!

That threshold value may be used for subsequent images, but it is best to look at the threshold for each image for verification, in the event of sample to sample differences. Be sure to save a reference image as a way to test day to day abilities at setting the threshold. For my own part, I cover the threshold value with my finger on the screen when moving the slider to see if I have come within 5 points of the same place. Normally, I find the same value or I'm off 1-3 points. This variation in the setting can be plotted to arrive at a standard error.

6. If necessary, fill all but the white objects with black. If you have selected an area of your image with the Lasso Tool at the beginning of these steps, you will want to invert the selection (in the menu under Select choose Inverse) and then fill with black (in the menu under Edit choose Fill: in that dialogue box, click arrowhead adjacent to Use and choose Black). In that manner, all visual information is black except for the objects. When desiring an area/area measurement, you will need to save two images for subsequent quantitation: one for measuring what you have outlined at the beginning and one for the objects themselves.

The entire process can be recorded using the Actions window (in the menu under Window select Show Actions). Click the arrowhead at the top right of the Actions dialogue box and choose New Action. Give the action a name. Simply proceed with all the steps listed above (be sure the image of interest is opened before recording), then click the black square at the bottom to stop the recording. Next to Threshold on the list of actions, click on the gray box to create a white box. That makes this step interactive. Click on the name of the action to highlight it, and click on the right pointing arrowhead at the bottom to test the action on a new image.

To automate these steps for a number of images, in the menu under File select Automate and then Batch. Set the Action setting to your newly named action, choose a folder with images to which you wish to apply your action, then be sure to make a folder as a destination for the segmented images (otherwise you save over your files never to get them back again).

Now that these objects are segmented, various measurements such as areas, counts, lengths, etc., can be found in your favorite quantitation program. The image may have to be inverted in the event that the quantitation program "sees" black (versus white objects) as the regions of interest. The smaller specks can be thrown away by filtering all objects at less than so many pixels (you be the judge): certainly all single pixels should be eliminated. Edge-touch- 


\section{IXRF Expands "Coast to Coast" celebrating their 10 year anniversary}

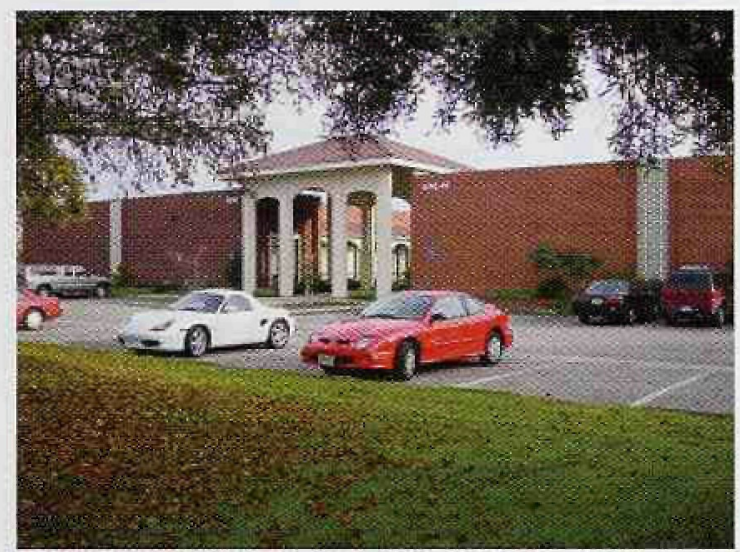

IXRF's Silicon Valley development facility

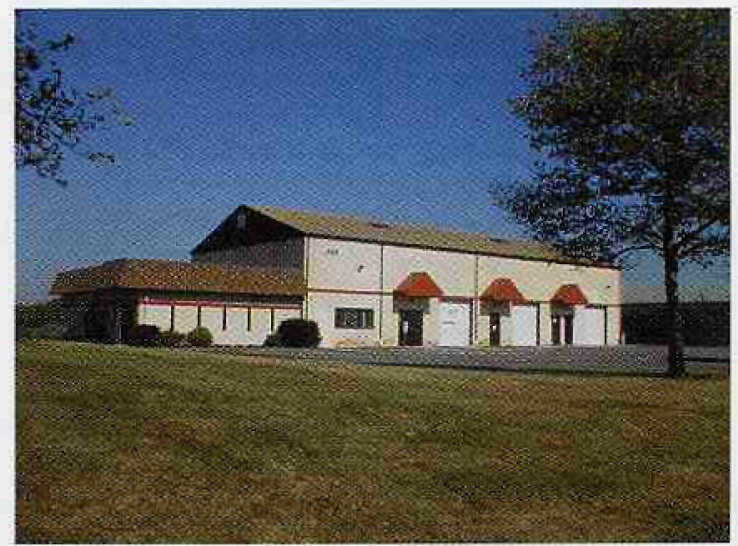

IXRF's Trenton New Jersey facility

IXRF thanks its loyal customers for their support in the growth of IXRF. At IXRF, we believe "satisfied" customers is the best way to sell more systems. By offering free software upgrades, customers never have to purchase another system. Even IXRF's first customers, 10 years ago, can still download the latest software and have all the latest features. Through customer suggestions, IXRF's software has become the leading Microanalysis software rivaling the major EDS companies -at a fraction of the cost. To our customers, thanks again!

Major Milestones

1992: IXRF personnel begin planning future products.

1993: Los Alamos National Lab receives the first IXRF system.

1995: Digital Imaging, Feature analysis, and X-Ray mapping are added into the systems.

1997: IXRF completes the first 100\% Integrated EDS Microanalysis system in history (developed for LEO Electron Microscopy).

1998: Jetscan Engine Health Monitor wins a millenium award from the British government (developed for LEO Electron Microscopy).

1999: IXRF Completes the "Particle Scan" offering the first truly integrated Particle Analysis inside the operating system of the SEM (developed for LEO Electron Microscopy).

1999: EXRF designs a new hardware interface allowing the use of Oxford PentaFET detectors for upgrading existing customers.

2000: IXRF completes the second fully Integrated EDS system, this time for JEOL Ltd.

2001: IXRF completes Particle Analysis on the Integrated JEOL Ltd product line.

2002: IXRF mounts the first micro x-ray tube on an SEM, to offer the first fully-integrated XRF and EDS microanalysis within the SEM.

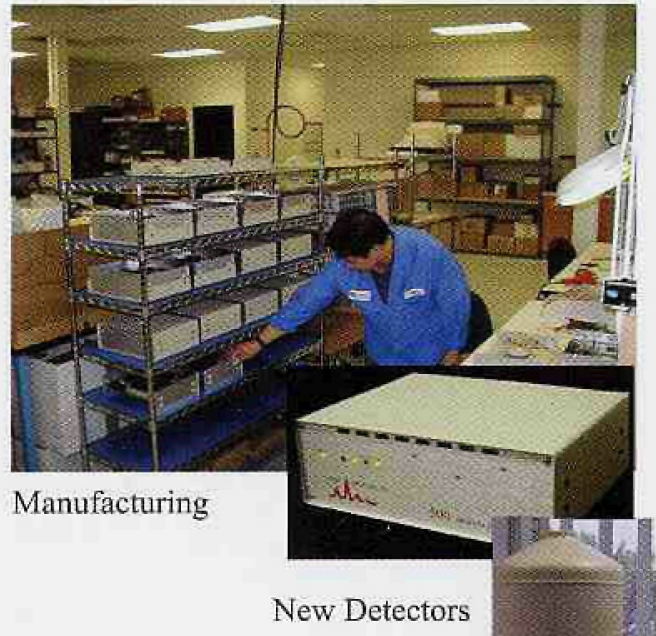

New Detectors

People who know X-Rays know IXRF!
IXRF Systems, Inc., Houston, TX, USA, www. ixrfsystems,com Ph:(281) 286-6485 Fax:(281)286-2660 


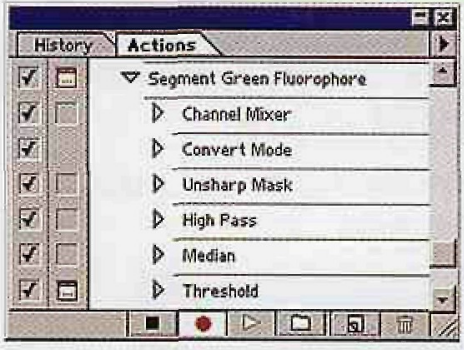

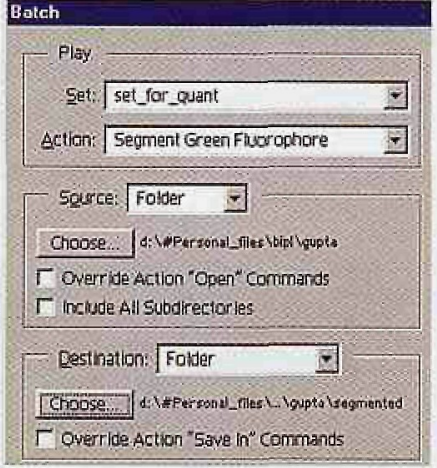

Figure 4. Examples of the Action dialogue box after recording a series of steps used for segmentation (above) and the Batch dialogue box (right)

ing objects can also be eliminated in many quantitation programs in the event that these are viewed as objects which are not positively identifiable. When it comes to automated counting, decisions can be made regarding how this should occur since objects often touch each other (some quantitation program search for an average size for an object and then divide the sum area of all objects, others rely upon binary images in which boolean filters are used, such as erode, dilate or watershed filters).

Often areas are found in Photoshop itself by selecting objects (this can be done by using Color Range under Select in the menu; in the dialogue box next to Select, click on the arrowhead to choose Highlights) and then looking at the area in pixels using Histogram (under Image on the menu). On some PC's, it is important to zoom in on the image so that the percentage zoom listed in the bar at the top of the image window reads $100 \%$. Because Photoshop appears to use the video card and monitor as a means for arriving at pixel sums rather than looking to the image file itself, one should be hesitant to use this means for arriving at measurements.

When desiring to measure intensities of fluorescing objects, obviously the black and white image cannot be used. You can, however, transfer the selection from the black and white image to the original image. Again, use Color Range as stated above to make your selection, and then, in the menu under Select, choose Save Selection. In the Save Selection dialogue box, it's a good idea to name the selection. On the original image choose Load Selection under Select on the menu and choose the selection from the black and white image (keep in mind that selections do not load when using two image files at different dimensions). Invert the selection on the original image and fill with black (as stated above). These steps-or similar steps-can also be accomplished in many quantitation programs in the event that 12- or 16-bit measurements are needed (remember that Photoshop requires 8-bit for many functions).

This file can now be thresholded easily in a quantitation program to identify the regions of interest for intensity measurements. Again, the mean pixel value can be found in Histogram within Photoshop, but the measurements appear to rely upon the video card/monitor rather than the inherent values in the image itself.

Adobe and Photoshop are registered trademarks of the Adobe Corporation, Mountain View, CA.

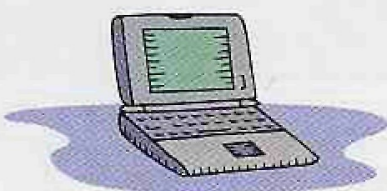

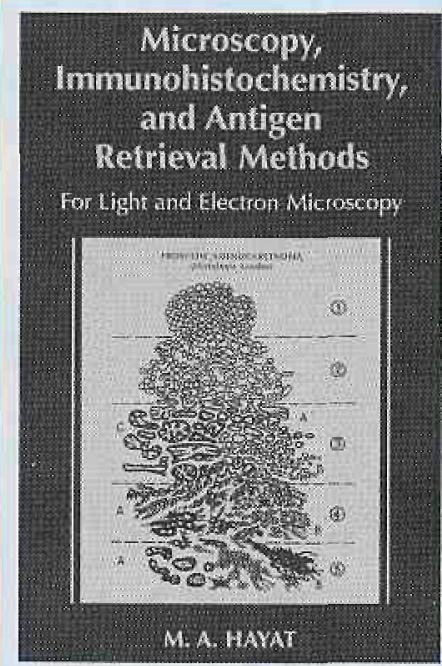

M.A. Hayat

Kean University, Union, NJ, USA

The most comprehensive methodology resource available in the field of immunohistochemistry of protein antigens and carbohydrate antigens. This important new work clearly presents chemical and physical principles governing the processing of tissues for histochemistry, immunohistochemistry, and immunocytochemistry and discusses both advantages and limitations of these methodologies. The book addresses the most recent developments in the retrieval and localization of antigens in normal and pathological tissues.

"A brilliantly explained text, containing extremely good applications, and above all, clear and well-referenced recipes. Most pitfalls in microscopy are the result of not following optimal processing conditions. After reading Dr. Hyat's present publication, there is no excuse for not following well-tested reliable protocols.

This Handbook is the most authoritative publication in the disciplines of microscopy, immunohistochemistry and antigen retrieval methods. It is expected to become $a$ classic in teaching and research. This book should not be absent in your personal collection, as well as the laboratory."

Prof. dr. E. Marani, Afdeling Fysiologie, Leiden. The Netherlands July 2002, Hardbound, ISBN 0-306-46770-4, 356 pp.

$\$ 110.00 / € 127.00 / \notin 78.00$

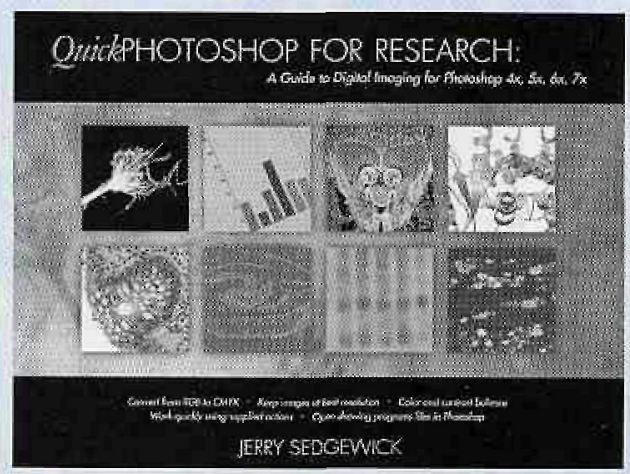

\section{Gerald Sedgewick}

Biomedical Image Processing Lab, University of Minnesota, MN, USA

Quick Photoshop for Research:A Guide to Digital Imaging contains essential information on the use of Photoshop specific to researchers. This step-by-step guide is the only book published for users whose needs are not for the purpose of graphic or web design: instead, this book only addresses the tools and functions necessary for the ethical enhancement of scientific images, and subsequent layout of these images into figures or plates. The aim is to provide information about digital imaging in an easy-to-follow guide from the beginning of the imaging process to its end.Additional information about scanning and acquiring images via a digital camera or laser/PMT system is also covered, as well as information about printers and PowerPoint.

"I strongly recommend this book in every research lab regularly working with publishing scientific artwork."

Hans de Jong, Laboratory of Genetics, Wageningen University, The Necherlands August 2002, Spiral-bound paperback, ISBN 0-306-47375-5, 120 pp. $\$ 49.50 / € 52.50 / € 35.00$

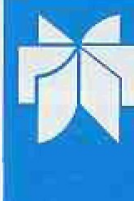

kluwer

the language of science Order online at www.wkap.com 


\section{DON'T OPERATE}

\section{BLINDFOLDED}

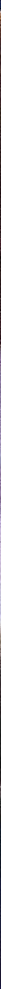

The LEO Crossbeam ${ }^{\oplus}-$ operates with simultaneous Field Emission SEM imaging and FIB milling.

Only with the IEO Crossbeam can you see your sample os your re milling it =

so therels no need to work bindfolded anymore. Crossbeam combines the CEMINI Field Emission adumn With proven ionbeam lechnolagy inio a single integratied foot The LEO Crossbeam is ideal for:

- Failure analysis

- TEM sample preparation

- Cross section investigation

- Three dimensional structural examination

Use the LEO Crossbeam and stop working blindfolded! Call 1-800-356-1090 today!

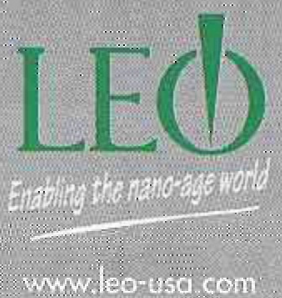

